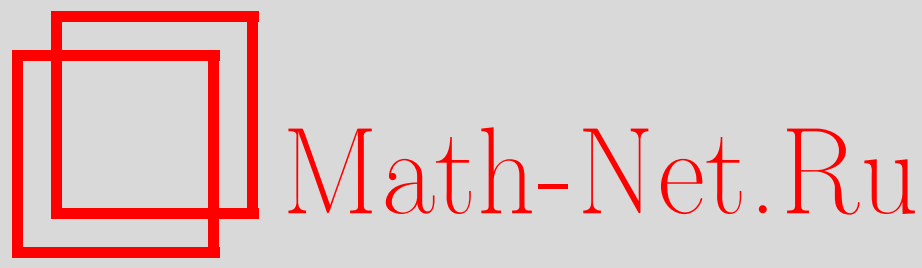

А. Сова, Квантовое запутывание в составных системах, ТМФ, 2009, том 159, номер 2, 283-298

DOI: https://doi.org/10.4213/tmf6349

Использование Общероссийского математического портала Math-Net.Ru подразумевает, что вы прочитали и согласны с пользовательским соглашением http://www . mathnet.ru/rus/agreement

Параметры загрузки:

IP: 35.174 .16 .151

26 апреля 2023 г., 14:12:14

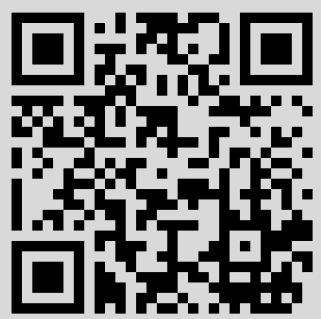




\title{
ФИЗИКА
}

Том 159, № 2

май, 2009

2009 г.

A. Сова*

\section{КВАНТОВОЕ ЗАПУТЫВАНИЕ В СОСТАВНЫХ СИСТЕМАХ}

\begin{abstract}
Рассматривается некоторый класс моделей мезоскопических квантовых явлений; эти модели возникают из сопоставления гамильтоновой динамики определенного типа и запутанных состояний составных систем. С помощью таких моделей можно получить точное описание феноменологии двумерного электронного газа, в особенности квантового эффекта Холла. Показано, как такие модели (если использовать их для отражения реальных физических принципов, а не только феноменологии) можно проверить в экспериментах типа квантовой телепортации. Так называемые мезоскопические модели предусматривают, что динамика взаимодействующего с внешним полем двумерного газа описывается функционалом типа $\operatorname{Tr}[H \rho]+\beta \operatorname{Tr} f(\rho)$, где $\rho$ - оператор плотности, а $H-$ одночастичный гамильтониан. На основе предлагаемого подхода показано, что с помощью подходящего эксперимента по квантовой телепортации можно получить информацию об аналитической функции $f$. Это приводит к взгляду на составную систему электронный газ-внешнее поле как на естественный квантовый компьютер.
\end{abstract}

Ключевые слова: составные системы, нелокальные модели, квантовое запутывание, квантовые эффекты Холла.

\section{1. КРАТКОЕ СОДЕРЖАНИЕ}

Мы будем рассматривать с новой точки зрения определенные теоретические концепции, которые исходно были введены в работах [1], [2] и затем применены в работах [3]-[5]. Наши методы можно использовать для моделирования систем, действующих в режиме квантового эффекта Холла (КЭХ) и его функциональной окрестности. Более того, такой подход, по-видимому, указывает на значимость определенных универсальных принципов при моделировании наносистем и в физике наносистем в целом. Основное новшество в используемом нами подходе состоит в использовании динамической переменной, которую можно рассматривать как квадратный корень из матрицы плотности. В настоящей работе мы подчеркиваем, что эта конструкция допускает интерпретацию в рамках квантовой теории информации, причем определяющую роль играет квантовое запутывание. Данный аспект рассматриваемой конструкции систематически обсуждается в настоящей работе впервые.

${ }^{*}$ Department of Mathematics and Statistics, University of Saskatchewan, Saskatoon, Canada. E-mail: sowa@math.usask.ca, a.sowa@mesoscopia.com 
Мы начнем с рассмотрения элементарного случая невзаимодействующего электронного газа, а затем перейдем к основному предмету исследования - к взаимодействующему газу. Это позволит интерпретировать квантовые системы Холла в рамках естественных квантовых вычислений. Наше рассмотрение не сильно ограничено тем предположением, что гильбертовы пространства и операторы конечномерны. Мы будем, вообще говоря, работать в этих рамках, а выходить за них будем только в тех случаях, когда это необходимо. Несколько исключений, когда нам потребуется прибегнуть к дифференциальным операторам и функциональным пространствам, будут оговорены особо.

\section{2. НЕВЗАИМОДЕЙСТВУЮЩИЕ СИСТЕМЫ: ЛИНЕЙНАЯ МЕХАНИКА}

\section{1. Базисное описание ансамбля невзаимодействующих электронов.} Начнем с обзора основ квантовой динамики. Дальнейшее рассмотрение будет основываться на хорошем понимании этих концепций. Наша основная конструкция существенна только для описания ансамбля электронов, которые не взаимодействуют между собой, а также не взаимодействуют с другими системами (с внешними полями). Пусть $\mathbf{H}-$ пространство, снабженное скалярным произведением $\langle\cdot \mid \cdot\rangle$. Динамика определяется гамильтонианом $H: \mathbf{H} \rightarrow \mathbf{H}$, который является эрмитовым линейным оператором, т.е. $H^{*}=H$. В частности, гамильтониан $H$ диагонализуем в базисе, состоящем из его собственных значений:

$$
H \psi_{k}=E_{k} \psi_{k} .
$$

Невзаимодействующую систему можно описывать волновой функцией $\psi(t, \cdot) \in \mathbf{H}$, которая удовлетворяет уравнению Шредингера

$$
i \dot{\psi}=H \psi .
$$

Среднее значение энергии системы в момент времени $t$ есть

$$
\langle H\rangle=\Lambda(\psi)=\langle\psi \mid H \psi\rangle .
$$

Это функционал, который порождает динамику в (2). Действительно, разбивая скалярное произведение на вещественную и мнимую части, получаем евклидово произведение и симплектическую форму:

$$
\langle X \mid Y\rangle=(X, Y)+i \omega(X, Y) .
$$

Можно вычислить производную равенства (3) следующим образом:

$$
\left.\frac{d}{d \varepsilon}\right|_{\varepsilon=0} \Lambda(\psi+\varepsilon \phi)=2 \operatorname{Re}\langle\phi \mid H \psi\rangle=2 \operatorname{Im}\langle i \phi \mid H \psi\rangle=2 \omega(\phi,-i H \psi) .
$$

В силу этих формул уравнение (2) по определению представляет собой гамильтонов поток, определяемый функцией Гамильтона (3) (см. монографию [6]). Заметим, что если $H$ не зависит от времени, то и среднее значение $\langle H\rangle$ заморожено во времени, хотя $\psi$ при этом эволюционирует. Действительно,

$$
\langle\dot{H}\rangle=\langle\dot{\psi} \mid H \psi\rangle+\langle H \psi \mid \dot{\psi}\rangle=\langle-i H \psi \mid H \psi\rangle+\langle H \psi \mid-i H \psi\rangle=0 .
$$


Альтернативное описание квантовой системы типа свободного электронного газа основано на использовании оператора плотности $\rho: \mathbf{H} \rightarrow \mathbf{H}, \rho^{*}=\rho$, удовлетворяющего, кроме того, условию $\operatorname{Tr} \rho=1$. Закон, определяющий эволюцию $\rho,-$ это уравнение движения Гейзенберга

$$
i \dot{\rho}=[H, \rho]:=H \rho-\rho H \text {. }
$$

Хорошо известен пример решения этого уравнения, который подчеркивает тот факт, что $\rho$-описание системы является обобщением $\psi$-описания. А именно, если выполнено (2), то оператор $\rho$, определяемый как $\rho=|\psi\rangle\langle\psi|$, удовлетворяет уравнению (6). Действительно,

$$
i \dot{\rho}=i|\dot{\psi}\rangle\langle\psi|+i| \psi\rangle\langle\dot{\psi}|=H| \psi\rangle\langle\psi|-| \psi\rangle\langle\psi| H=[H, \rho] .
$$

Более того, если $\psi=\sum_{k} a_{k} \psi_{k}$, то $\rho=\sum_{k, l} a_{k} \bar{a}_{l}\left|\psi_{k}\right\rangle\left\langle\psi_{l}\right|$ и $H \rho=\sum_{k, l} E_{k} a_{k} \bar{a}_{l}\left|\psi_{k}\right\rangle\left\langle\psi_{l}\right|$. Поэтому

$$
\langle H\rangle=\langle\psi \mid H \psi\rangle=\sum_{k}\left|a_{k}\right|^{2}\left\langle\psi_{k} \mid H \psi_{k}\right\rangle=\sum_{k}\left|a_{k}\right|^{2} E_{k}\left\langle\psi_{k} \mid \psi_{k}\right\rangle=\operatorname{Tr}(H \rho) .
$$

Стандартное скалярное произведение в пространстве конечномерных комплексных операторов имеет вид

$$
\langle A \mid B\rangle=\operatorname{Tr}\left(A B^{*}\right) .
$$

Это дает основание определить среднее значение энергии в $\rho$-описании следующим образом:

$$
\langle H\rangle=\langle H \mid \rho\rangle .
$$

Такое скалярное произведение играет важную роль в дальнейшем рассмотрении. Напомним следующие фундаментальные тождества:

$$
\langle M A \mid B\rangle=\left\langle A \mid M^{*} B\right\rangle, \quad\langle A M \mid B\rangle=\left\langle A \mid B M^{*}\right\rangle, \quad\langle[A, M] \mid B\rangle=\left\langle A \mid\left[B, M^{*}\right]\right\rangle,
$$

которые выполнены для произвольных $A, B$ и $M$. В силу последних тождеств и уравнения (6) из независимости $H$ от времени следует, что $\langle H\rangle$ также не зависит от времени в $\rho$-описании.

И последнее по порядку, но не по важности: подчеркнем, что скалярное произведение $(7)$ индуцирует евклидово произведение $(\cdot, \cdot)$ и симплектическую форму $\omega(\cdot, \cdot)$ в соответствии с (4) на пространстве конечномерных комплексных операторов.

\section{2. “Квадратный корень” из матрицы плотности как динамическая пе-} ременная. Согласно уравнению (6) оператор плотности $\rho$ является динамической переменной, но встает вопрос, существует ли вариационный функционал, содержащий $\rho$, из которого можно было бы получить данное уравнение движения? Было бы естественным спросить, должна ли сама формула (8) определять динамическую переменную $\rho$ ? В конце концов, она описывает среднее значение энергии, как и (3). Однако выражение (8) линейно по $\rho$, и поэтому его градиент равен $\langle H|$. Таким образом, этого выражения самого по себе не достаточно для определения динамики (6). 
Однако картина изменится, если мы представим $\rho$ в виде произведения двух комплексных и, как правило, неэрмитовых операторов, т.е. в виде $\rho=K^{*} K$. Заметим, что это указывает на возможность введения еще одного гильбертова пространства, дополнительного к Н. Будем обозначать его через $\widehat{\mathbf{H}}$. Действительно, если

$$
K: \mathbf{H} \rightarrow \widehat{\mathbf{H}},
$$

то в этом случае по-прежнему

$$
\rho=K^{*} K: \mathbf{H} \rightarrow \mathbf{H}
$$

В последующих разделах мы рассмотрим роль этого дополнительного элемента конструкции. Подчеркнем, что представление $\rho$ через $K$ не единственно, поскольку при произвольном унитарном $U$ взятие $U K$ вместо $K$ дает то же $\rho$.

Подставляя (10) в (8), получаем нетривиальный гамильтониан

$$
\Lambda(K)=\langle H\rangle=\left\langle H \mid K^{*} K\right\rangle=\operatorname{Tr}\left(H K^{*} K\right)=\operatorname{Tr}\left(K H K^{*}\right)=\langle K H \mid K\rangle .
$$

Вычисление, аналогичное (5), дает

$$
\left.\frac{d}{d \varepsilon}\right|_{\varepsilon=0} \Lambda(K+\varepsilon \Phi)=2 \operatorname{Re}\langle\Phi \mid K H\rangle=2 \operatorname{Im}\langle\Phi \mid-i K H\rangle=2 \omega(\Phi,-i K H),
$$

где теперь $\Phi$ - комплексный оператор. Применяя правила гамильтоновой механики, но обращая направление течения времени (по причинам, которые скоро прояснятся), получаем уравнение

$$
-i \dot{K}=K H
$$

откуда автоматически следует

$$
i \dot{K}^{*}=H K^{*} .
$$

Заметим, что таким образом столбцы оператора $K^{*}$ удовлетворяют исходному уравнению Шредингера (2). Кроме того, если $K$ удовлетворяет этому уравнению, то $\rho=K^{*} K$ получается из своей канонической динамики. Действительно,

$$
i \dot{\rho}=i \dot{K}^{*} K+i K^{*} \dot{K}=H K^{*} K-K^{*} K H=[H, \rho] .
$$

При этом если оператор $K K^{*}$ заморожен во времени, то получим

$$
i\left(K \dot{K}^{*}\right)=i \dot{K} K^{*}+i K \dot{K}^{*}=-K H K^{*}+K H K^{*}=0 .
$$

Таким образом, уравнение (12), или, что эквивалентно, уравнение (13) воспроизводит квантово-механическую эволюцию оператора плотности $\rho$. В то же время, вводя оператор $K$, мы смогли включить функционал (8) в динамическую картину с самого начала. Кроме того, описание динамики электронного газа на основе оператора $K$ позволяет рассматривать кросс-корреляции в смеси чистых состояний. Например, оператор плотности $\rho=\sum_{k} c_{k}\left|\psi_{k}\right\rangle\left\langle\psi_{k}\right|$ может соответствовать оператору $K=\sum_{k} \sqrt{c_{k}} e^{i \theta_{k}}\left|\psi_{k}\right\rangle\left\langle\psi_{k}\right|$, в силу чего чистым состояниям $\left|\psi_{k}\right\rangle\left\langle\psi_{k}\right|$ явным образом приписаны фазы. Информация о фазах теряется, если вернуться к $\rho$-описанию ансамбля состояний. 
2.3. Интерпретация $K$ в терминах запутывания. Введение оператора (9) допускает естественную интерпретацию на основе теории квантовых измерений и квантовой информации [7]. Сначала выразим оператор $K$ в общем виде $K=$ $\sum A_{m n}\left|\varphi_{m}\right\rangle\left\langle\psi_{n}\right|$, где $\left\{\left|\varphi_{m}\right\rangle\right\}$ - ортонормированный набор состояний из $\widehat{\mathbf{H}}$. Заметим, что, поскольку $K^{*} K$ представляет собой оператор плотности, мы имеем

$$
\sum_{m, n}\left|A_{m n}\right|^{2}=\|K\|^{2}=\operatorname{Tr}\left(K K^{*}\right)=\operatorname{Tr}\left(K^{*} K\right)=1 .
$$

Далее, преобразуем представление для $K$ следующим образом:

$$
K=\sum_{m}\left|\varphi_{m}\right\rangle \sum_{n} A_{m n}\left\langle\psi_{n}\left|=\sum_{m}\right| \varphi_{m}\right\rangle\left\langle w_{m}\right|, \quad\left\langle w_{m}\right|=\sum_{n} A_{m n}\left\langle\psi_{n}\right| .
$$

Заметим, что введение оператора $K$ эквивалентно введению состояния

$$
\left|\Psi_{\mathrm{comb}}\right\rangle=\sum_{m, n} A_{m n}^{*}\left|\varphi_{m}\right\rangle \otimes\left|\psi_{n}\right\rangle=\sum_{m}\left|\varphi_{m}\right\rangle \otimes\left|w_{m}\right\rangle \in \widehat{\mathbf{H}} \otimes \mathbf{H},
$$

которое является одним из возможных состояний системы, составленной из подсистем Н и $\widehat{\mathbf{H}}$. Заметим, что в силу (16) мы автоматически имеем $\left\|\Psi_{\text {comb }}\right\|=1$. Это приводит к следующей интерпретации [7]: после наблюдения состояния $\left|\varphi_{m}\right\rangle$ в системе $\hat{\mathbf{H}}$, система $\mathbf{H}$ с вероятностью единица окажется в коррелированном состоянии $\left|w_{m}\right\rangle$. Заметим, что

$$
\left|w_{m}\right\rangle=K^{*}\left|\varphi_{m}\right\rangle
$$

Конечно, две системы могут поменяться ролями. Действительно, можно представить оператор $K$ в дуальном виде:

$$
K=\sum_{n}\left(\sum_{m} A_{m n}\left|\varphi_{m}\right\rangle\right)\left\langle\psi_{n}\left|=\sum_{n}\right| u_{n}\right\rangle\left\langle\psi_{n}\right|,
$$

где последнее равенство определяет $\left|u_{n}\right\rangle$. Теперь после наблюдения состояния $\psi_{n}$ в системе Н система $\widehat{\mathbf{H}}$ окажется в состоянии $\left|u_{n}\right\rangle$, где

$$
\left|u_{n}\right\rangle=K\left|\psi_{n}\right\rangle
$$

Соотношения (19) и (21) позволяют интерпретировать $K$ как оператор, который определяет корреляции между измерениями над системами $\mathbf{H}$ и $\widehat{\mathbf{H}}$. Мы иногда будем называть $K$ оператором запутывания.

Напомним, что состояние $\left|\Psi_{\text {соть }}\right\rangle$ называется незапутанным, если его можно записать в виде $\left|\Psi_{\text {соть }}\right\rangle=|\Phi\rangle \otimes|\Psi\rangle$. Легко видеть, что $\left|\Psi_{\text {соть }}\right\rangle$ незапутанно, если и только если $\rho$, определяемый в $(10)$, является проектором на одномерное подпространство. В самом деле, последнее условие эквивалентным образом выражается как наличие такого состояния $|\Psi\rangle$, что $\rho=|\Psi\rangle\langle\Psi|$.

Наконец, заметим, что представление $\rho$ в виде (10) эквивалентно тому, что

$$
\rho=\operatorname{Tr}_{\widehat{\mathbf{H}}}\left(\left|\Psi_{\mathrm{comb}}\right\rangle\left\langle\Psi_{\mathrm{comb}}\right|\right)=\sum_{m}\left|w_{m}\right\rangle\left\langle w_{m}\right|
$$


поскольку в силу соотношения (17) и ортонормированности $\left\{\left|\varphi_{m}\right\rangle\right\}$ мы имеем $K^{*} K=$ $\sum_{m}\left|w_{m}\right\rangle\left\langle w_{m}\right|$. Выражение (22) дает канонический способ вычисления редуцированного оператора плотности подсистемы, а задание $\rho$ в виде (10) является при этом альтернативой, которая имеет свои преимущества. В частности, мы увидим, что явное использование оператора $K$ позволяет применить методы гамильтоновой динамики в нестандартном описании запутанных квантовых систем.

\section{3. ВЗАИМОДЕЙСТВУЮЩИЕ СИСТЕМЫ. НЕЛИНЕЙНАЯ МЕХАНИКА}

3.1. Подход к описанию взаимодействующих электронов. До сих пор мы описывали эволюцию ансамбля невзаимодействующих частиц. Теперь попытаемся учесть взаимодействие между частицами путем следующей модификации полной энергии: $\langle H\rangle=\langle H \mid \rho\rangle+F(\rho)$. Здесь член с дополнительной энергией не должен зависеть от $H$ и от конкретного матричного представления оператора плотности $\rho$. Мы рассмотрим достаточно общие способы выбора $F$ в разделе 5 , но сначала обратимся к специальному случаю

$$
F(\rho)=\beta \ln \operatorname{det} \rho,
$$

где $\beta$ - константа. Заметим, что данный случай связан с относительной квантовой энтропией [7]. Напомним, что хорошо известная энтропия фон Неймана определяется для системы как

$$
S(\rho)=-\operatorname{Tr}(\rho \ln \rho),
$$

с другой стороны, относительная энтропия определяется с помощью двух матрищ плотности следующим образом:

$$
S\left(\rho^{0}, \rho\right)=\operatorname{Tr}\left(\rho^{0} \ln \rho^{0}\right)-\operatorname{Tr}\left(\rho^{0} \ln \rho\right) .
$$

Эти соотношения квантового типа, разумеется, аналогичны известным формулам, играющим центральную роль в теории информации Шеннона. Однако их интерпретация установлена не столь хорошо, как для их классических аналогов. Вспомним теперь известную формулу $\operatorname{det} e^{A}=e^{\operatorname{Tr} A}$. Из нее следует, что функционал (23) имеет смысл относительной энтропии:

$$
\ln \operatorname{det} \rho=\operatorname{Tr} \ln \rho=N S_{0}-N S(\bar{I}, \rho) .
$$

Здесь $\bar{I}=N^{-1} I$ - перенормированный единичный оператор, так что $\operatorname{Tr} \bar{I}=1$, а $S_{0}-$ константа, которая не зависит от $\rho$. Можно отметить, что выбор $\rho^{0}=\bar{I}$ выделяет "белый шум" среди других состояний.

Далее мы рассмотрим описание взаимодействующего электронного газа на основе функционала $\operatorname{Tr}(H \rho)+\beta \ln \operatorname{det} \rho$ среднего значения энергии. Нас интересует ситуация, когда определитель не равен нулю, поэтому мы полагаем, что $\rho>0$. В частности, собственные векторы оператора $\rho$ линейно порождают все пространство Н (это допущение будет изменено при рассмотрении бесконечномерного случая). Чтобы получить осмысленную динамику, мы прибегнем к подходу, введенному в предыдущем разделе, т.е. представим эрмитову матрицу $\rho$ как композицию комплексных 
матриц, как в (10), и изучим функционал

$$
\Xi(K)=\operatorname{Tr}\left(K H K^{*}\right)+\beta \ln \operatorname{det}\left(K^{*} K\right),
$$

который будет находиться в центре нашего внимания в оставшейся части статьи. Его обобщения будут рассмотрены в разделе 5 .

\section{2. Производная функционала $\Xi$ и соответствующая гамильтонова ди-} намика. Чтобы изучить динамику, индуцируемую функционалом (26), надо вычислить производную определителя. Элементарное (хотя и нетривиальное) вычисление показывает, что

$$
\left.\frac{d}{d \varepsilon}\right|_{\varepsilon=0} \ln \operatorname{det}(K+\varepsilon \Phi)=\left\langle\Phi \mid K^{*-1}\right\rangle
$$

и аналогично

$$
\left.\frac{d}{d \varepsilon}\right|_{\varepsilon=0} \ln \operatorname{det}\left(K^{*}+\varepsilon \Phi^{*}\right)=\operatorname{Tr}\left(K^{*-1} \Phi^{*}\right)=\left\langle K^{*-1} \mid \Phi\right\rangle .
$$

Эти формулы можно сравнить с результатами вычислений производной функционала такого типа в более общем случае (см. раздел 5). Далее, вычисление, аналогичное (11), дает

$$
\left.\frac{d}{d \varepsilon}\right|_{\varepsilon=0} \ln \operatorname{det}\left(\left(K^{*}+\varepsilon \Phi^{*}\right)(K+\varepsilon \Phi)\right)=2 \operatorname{Re}\left\langle\Phi \mid K^{*-1}\right\rangle=2 \omega\left(\Phi,-i K^{*-1}\right) .
$$

Таким образом, функционал $\Xi$, определенный в (26), приводит к гамильтонову эволюционному уравнению

$$
-i \dot{K}=K H+\beta K^{*-1} .
$$

Мы будем иногда называть это уравнение мезоскопическим уравнением Шредингера или просто мезоскопическим уравнением. Заметим, что оно представляет собой нелинейное обобщение уравнения (12), а сопряженное уравнение (13) также имеет аналог $i \dot{K}^{*}=H K^{*}+\beta K^{-1}$. Обратим внимание на то, что, хотя $K$ удовлетворяет нелинейному уравнению, $\rho$ по-прежнему удовлетворяет уравнению движения Гейзенберга. Действительно, видоизменяя надлежащим образом вычисления, приведенные в (14), получаем

$$
i \dot{\rho}=i \dot{K}^{*} K+i K^{*} \dot{K}=H K^{*} K+\beta K^{-1} K-K^{*} K H-\beta K^{*} K^{*-1}=[H, \rho] .
$$

Этот результат может несколько озадачить, поскольку он означает, что некто, намеревающийся следить только за эволюцией $\rho$ в системе, определенной функционалом (26), может не знать, что функционал энергии был изменен за счет слагаемого с относительной энтропией. Однако не будем забывать, что $K$ кодирует квантовое запутывание электронной подсистемы $\mathbf{H}$ с подсистемой $\widehat{\mathbf{H}}$. Эволюция оператора $K$ отчасти управляет поведением всей составной системы, в то время как только ее часть доступна для $\rho$. Ввиду этого представляется естественным рассматривать более богатую теорию, где система $\widehat{\mathbf{H}}$ может влиять на эволюцию оператора $\rho$ посредством гамильтониана $H$. Мы рассмотрим этот сценарий в разделе 4 . Упомянем также, что, вообще говоря, уравнение (29) более не выполнено, если допускать относительную энтропию (25) с произвольным $\rho^{0}$.

5 Теоретическая и математическая физика, т. 159, № 2, 2009 г. 
3.3. Собственные состояния как следствие нелинейности. Ненадолго вернемся к линейной динамике (12). Ясно, что нельзя использовать это уравнение для определения собственных состояний. В то же время можно определить их как стационарные решения уравнений Гейзенберга (6): стационарные решения - это те, которые коммутируют с $H$ (диагонализуются в собственном базисе оператора $H$ ). В то же время уравнение (28) позволяет указать другой вид собственных состояний непосредственно через оператор $K$. Действительно, подставляя $e^{i t \nu} K(x)$ вместо $K(t, x)$, получаем стационарное уравнение

$$
K H+\beta K^{*-1}=\nu K .
$$

Отсюда следует, что

$$
\rho=K^{*} K=\beta(\nu I-H)^{-1} .
$$

Собственные состояния имеют вид

$$
K=U\left(\frac{1}{\beta}(\nu I-H)\right)^{-1 / 2},
$$

где $U: \mathbf{H} \rightarrow \widehat{\mathbf{H}}-$ произвольный унитарный оператор. Как и раньше, соответствующие собственным состояниям операторы $\rho$ диагонализуются одновременно с $H$. Заметим, что для существования решения величина $\nu$ должна превосходить все собственные значения оператора $H$. Уравнению (31) можно придать смысл, даже когда $H$ - дифференциальный оператор [2], просто сужая область определения $\rho$. С учетом (1) получаем

$$
\rho=\beta \sum_{E_{k}<\nu} \frac{1}{\nu-E_{k}}\left|\psi_{k}\right\rangle\left\langle\psi_{k}\right| .
$$

Константу $\beta$ следует выбрать так, чтобы оператор $\rho$ был правильно нормирован. Собственные состояния являются смесями конечного числа чистых состояний. Более того, ингредиенты присутствуют в определенной пропорции. Это напоминает электронные свойства кристалла, где локализованные состояния находятся ниже уровня энергии Ферми. В частности, свойства $\rho$ зависят от конкретных значений $\nu$ и от размера щели между этим уровнем и следующим наивысшим занятым уровнем.

3.4. Стационарные корреляции между наблюдениями над Н и $\widehat{\mathbf{H}}$. Перепишем собственное состояние $K$ из (32) в виде, сходном с (33):

$$
K=\sum_{E_{k}<\nu}\left(\frac{\beta}{\nu-E_{k}}\right)^{1 / 2}\left|U \psi_{k}\right\rangle\left\langle\psi_{k}\right| .
$$

Согласно интерпретации $K$ в терминах запутывания это означает, что собственное состояние индуцирует корреляцию между наблюдениями над системами $\mathbf{H}$ и $\widehat{\mathbf{H}}$. Если первая из этих систем наблюдается в состоянии $\left|\psi_{k}\right\rangle$, то вторая коллапсирует к состоянию $\left|U \psi_{k}\right\rangle$ и наоборот. Поскольку $\left|\psi_{k}\right\rangle$ - собственное состояние гамильтониана, наблюдение этого состояния в системе $\mathbf{H}$ сопровождается измерением энергии или любой другой инвариантной по времени наблюдаемой. С другой стороны, наблюдение состояния $\left|U \psi_{k}\right\rangle$ связано с измерением наблюдаемой $U H U^{*}: \widehat{\mathbf{H}} \rightarrow \widehat{\mathbf{H}}$ или 


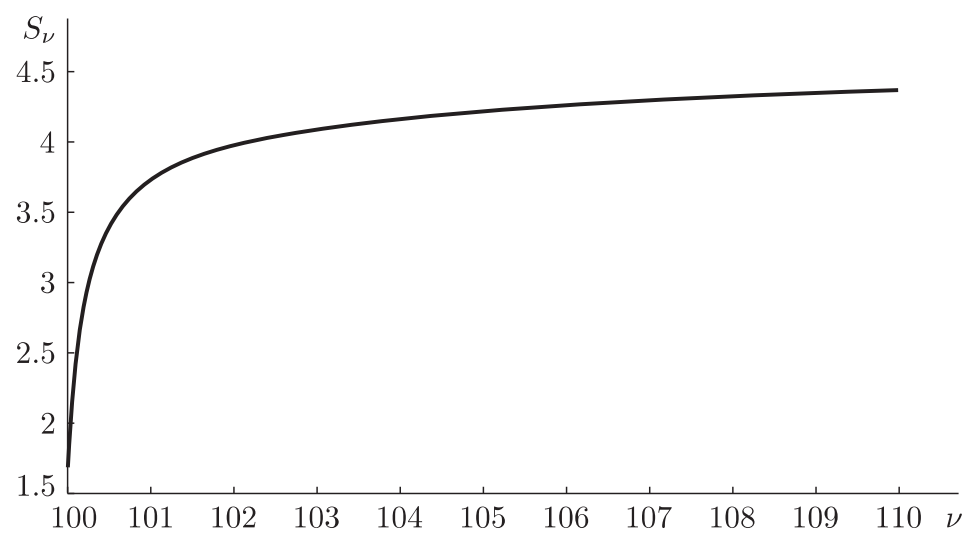

График $S_{\nu}$ из $(39)$ для модели со 100 случайными уровнями энергии в интервале $[1,100)$ при $\nu \in(100,110)$.

любой наблюдаемой, коммутирующей с ней. Поскольку оператор $U: \mathbf{H} \rightarrow \widehat{\mathbf{H}}$ произволен, он может отобразить базис $\left|\psi_{k}\right\rangle$ на любой базис в $\widehat{\mathbf{H}}$. Таким образом, $K$ не налагает никаких априорных ограничений на систему $\widehat{\mathbf{H}}$ в состоянии равновесия, соответствующем собственным состояниям.

Итерпретируя в терминах запутывания энтропию фон Неймана (24), можно сказать, что для $\rho=K^{*} K$ она является мерой запутывания соответствующего состояния (18). Когда система гомеостатична, так что матрица плотности имеет вид (33), запутывание есть

$$
S_{\nu}=-\sum_{E_{k}<\nu} \frac{\beta}{\nu-E_{k}} \ln \left(\frac{\beta}{\nu-E_{k}}\right) .
$$

Рисунок иллюстрирует типичную зависимость запутывания $S_{\nu}$ от $\nu$.

3.5. Корреляции в ходе временно́й эволюции. Следует подчеркнуть, что мезоскопическое уравнение (28) в некотором смысле совместно с квантовой механикой. Это заключение основано на том неожиданном наблюдении, что уравнение (28) можно решить почти явно в том смысле, что его решения можно выразить через решения линейного уравнения Шредингера [2]. На самом деле, это можно сделать, даже когда пространства $\mathbf{H}$ и $\widehat{\mathbf{H}}$ бесконечномерны. Рассмотрим динамическую переменную $K$ вида

$$
K(t)=\sum A_{m n}(t)\left|\varphi_{m}(t)\right\rangle\left\langle\psi_{n}(t)\right|,
$$

где сумма по индексам $m$ и $n$ в действительности конечна, скажем $m, n=1, \ldots, N$, но волновые функции $\left|\psi_{n}(t)\right\rangle$ и $\left|\varphi_{m}(t)\right\rangle$ обитают в бесконечномерных функциональных пространствах $\mathbf{H}$ и $\widehat{\mathbf{H}}$. Напомним, что для рассмотрения мезоскопического уравнения (28) нам пришлось предположить, что матрица $K$ несингулярна. Если мы рассматриваем данное уравнение в бесконечномерном случае, это условие следует заменить на требование обратимости матрицы $A=\left[A_{m n}\right]$. Другими словами, мы предполагаем, что $K(t): F(t) \rightarrow G(t)$ обратимо как отображение, имеющее суженные область определения и область значений

$$
F(t)=\operatorname{span}\left\{\left|\psi_{n}(t)\right\rangle, n=1, \ldots, N\right\}
$$


и

$$
G(t)=G(0)=\operatorname{span}\left\{\left|\varphi_{m}(0)\right\rangle, m=1, \ldots, N\right\} .
$$

Тогда ясно, как интерпретировать уравнение (28). Более того, оказывается, что решения характеризуются следующими тремя условиями:

1) образ остается замороженным во времени, $G(t)=G(0)$, и $\left|\varphi_{m}(t)\right\rangle=\left|\varphi_{m}(0)\right\rangle=$ $\left|\varphi_{m}\right\rangle$;

2) существует набор векторов $\left\{\psi_{n}(t)\right\}_{n=1, \ldots, N}$, который является унитарным базисом для изменяющейся со временем области определения $F(t)$ и, более того, все эти векторы удовлетворяют уравнению Шредингера

$$
i \dot{\psi}_{n}=H \psi_{n}
$$

3) изменяя ортонормированное семейство $\left\{\left|\varphi_{m}\right\rangle\right\}_{n=1, \ldots, N}$, можно получить самосопряженную матрицу $A(0)=A(0)^{*}$, при этом $A(t)$ определяется из начального условия по формуле

$$
A(t)=A(0) \exp \left(i \int_{0}^{t} \beta(\tau) A(0)^{-2} d \tau\right) .
$$

Кроме того, решения мезоскопического уравнения обладают тем свойством, что энергия $\Xi(K(t))$ инвариантна по времени, коль скоро $H$ и $\beta$ не зависят от времени [2], т.е. систему можно снабжать энергией только через составные элементы уравнения (28).

Таким образом, интерпретация $K$ в терминах запутывания означает, что если система Н обнаружена в состоянии $|\Psi\rangle$, то система $\widehat{\mathbf{H}}$ с вероятностью единица коллапсирует к состоянию

$$
|\Phi\rangle=K|\Psi\rangle=\sum A_{m n}(t)\left\langle\psi_{n}(t) \mid \Psi\right\rangle\left|\varphi_{m}\right\rangle
$$

Поэтому состояние, к которому коллапсирует подсистема $\widehat{\mathbf{H}}$, зависит не только от исхода эксперимента в системе $\mathbf{H}$, но и от момента времени. Более того, оно определяется шредингеровской эволюцией в $\mathbf{H}$, равно как и эволюцией (38) неабелева фазового множителя $A(t)$.

\section{4. КЭХ И КВАНТОВАЯ ТЕЛЕПОРТАЦИЯ}

4.1. Замыкание петли взаимодействия в составных системах. Рассмотрим $K: \mathbf{H} \rightarrow \widehat{\mathbf{H}}$, как и выше, в контексте двумерного электронного газа, взаимодействующего с магнитным полем. Тем самым мы интерпретируем $\mathbf{H}$ как квантовый дескриптор электронного газа, а $\widehat{\mathbf{H}}$ - как квантовый дескриптор внешнего магнитного поля. Мы полагаем, что системы электронного газа и магнитного поля запутаны. В соответствии с интерпретацией в терминах запутывания, если подсистема Н обнаружена в состоянии $|\Psi\rangle$, то подсистема $\widehat{\mathbf{H}}$ автоматически коллапсирует к состоянию $K|\Psi\rangle$. Это можно воспринимать как влияние электронной подсистемы на внешнее поле. Параллельно с этим рассмотрим описание электромагнитного поля с помощью классических уравнений поля. Это нужно для того, чтобы учесть влияние, которое электромагнитное поле оказывает на динамику электронного газа. 
А именно, классический дескриптор поля, т.е. векторный потенциал $A$, входит в гамильтониан $H=H_{A}=\nabla_{A}^{*} \nabla_{A}$, определяющий динамику $K$ посредством (26) (естественно, при этом надо рассматривать гамильтониан как дифференциальный оператор), и этим замыкается петля взаимодействия между электронным газом и электромагнитным полем. Заметим, что наш подход асимметричен по отношению к $\mathbf{H}$ и $\widehat{\mathbf{H}}$ - таковым он и был в начальный момент. Сначала обсудим, как этот тип петли обратной связи помогает описывать динамику сложных квантовых систем, а затем предложим тест для проверки этих принципов.

4.2. Квантовые системы Холла как составные системы. Стандартная модель взаимодействия электронов с внешним электромагнитным полем задается связанной системой уравнений Максвелла и Шредингера. Взаимодействие происходит через заряд и/или ток в соответствии с тем, как эти понятия можно интерпретировать в рамках и электромагнетизма, и квантовой механики. Однако этот подход теряет адекватность при описании таких более сложных явлений, как КЭХ. В то же время основную черту КЭХ - квантование холловского сопротивления - можно вывести из фундаментальных принципов квантовой теории; подходящие аргументы были основаны на релятивистской квантовой теории поля [8]. Здесь же мы кратко опишем другой подход к KЭХ [4], [5], основанный на понятии относительной энтропии и на гамильтоновом подходе к динамике, представленной в предыдущих разделах. Подчеркнем, что получающееся описание является нерелятивистским.

Рассмотрим собственные состояния $K(34)$ при дополнительном допущении $U=I$. Предположим, что наша система в достаточной мере описывается всего одной волновой функцией $\psi$ и гомеостатична, т.е. $\psi=\psi_{n_{0}}$, где $\psi_{n_{0}}-$ собственное состояние оператора $H$. Как предлагалось выше, мы хотим, чтобы обратная связь, идущая от электронной системы $\mathbf{H}$ к системе внешнего магнитного поля $\widehat{\mathbf{H}}$, определялась преобразованием запутывания $K$. Таким образом, состояние внешней системы имеет вид

$$
K \psi=K \psi_{n_{0}}=c \psi_{n_{0}}=c \psi,
$$

где $c$ - константа. В этом описании плотность потока определяется как

$$
B \sim|K| \psi\rangle\left.\right|^{2}=|c|^{2}|\psi|^{2}=R_{H}|\psi|^{2} .
$$

Заметим, что в общем случае пространственное распределение плотности потока неравномерно. Поскольку константа равна отношению полного потока к полному заряду, она обратна так называемой степени заполнения; мы обозначили ее как $R_{H}$. Далее, получающееся магнитное поле изменяет гамильтониан $H_{A}=\nabla_{A}^{*} \nabla_{A}$, где $A-$ векторный потенциал, $* d A=B$.

Петля взаимодействия описывается следующей системой уравнений:

$$
* d A=R_{H}|\psi|^{2}, \quad \nabla_{A}^{*} \nabla_{A} \psi=E \psi .
$$

В работе автора [3] показано, что эта модель предсказывает, что холловское сопротивление равно $R_{H}$, и это в действительности наблюдается в КЭХ. Отличительной чертой данной модели является ее конструктивность и возможность компьютерного моделирования. Более того, она позволяет явно изучать последствия возмущения модели различными способами, так, в работе автора [4] с определенной точки зрения проанализированы некоторые эффекты, следующие из расширения модели за 
счет решеточного потенциала. Мы предвидим возможность использовать различные возмущения этой модели и ее разнообразные расширения для сбора информации от систем, которые действуют вблизи режима КЭХ. При том что такие системы являются не более чем частным случаем наносистем, многие из положенных в их основу принципов представляются вполне универсальными.

\section{3. Эксперимент по квантовой телепортации как проверка принципа.}

Интересно спросить, может ли представленная выше модель в действительности дать адекватное описание рассматриваемой квантово-механической системы? Может ли она в действительности отражать не только феноменологию, но и принцип действия? Опишем в общих чертах, как эту гипотезу можно проверить в (идеализированном) эксперименте. Предположим, что составная система $\widehat{\mathbf{H}} \otimes \mathbf{H}$ описывается статическим оператором запутывания $K$, скажем

$$
K=\sum_{m, n=1}^{N} A_{m n}\left|\varphi_{m}\right\rangle\left\langle\psi_{n}\right|,
$$

где $\left|\psi_{n}\right\rangle \in \mathbf{H}$ и $\left|\varphi_{m}\right\rangle \in \widehat{\mathbf{H}}-$ взаимно ортогональные векторы с единичной нормой, а $A$ - несингулярная матрица. Мы хотим экспериментально проверить, удовлетворяет ли $K$ равенству (34). Для этого мы ставим эксперимент типа квантовой телепортации. Пусть А представляет внешний аппарат, предназначенный для измерения некоторой наблюдаемой с базисом из собственных векторов $\left|\alpha_{k}\right\rangle, k=1, \ldots, N$. Более того, переименуем $\left|\varphi_{m}\right\rangle$ и будем называть их $\left|\alpha_{m}\right\rangle$, что представляет собой просто формальную трансляцию квантовых алфавитов.

Пусть имеются два наблюдателя, Алиса и Боб. В некоторый момент времени Алиса производит наблюдение над $\mathbf{H} \otimes \mathbf{A}$, а вскоре после этого Боб производит наблюдение над $\widehat{\mathbf{H}}$. До эксперимента система $\widehat{\mathbf{H}} \otimes \mathbf{H} \otimes \mathbf{A}$ находилась, скажем, в состоянии

$$
\left|\Psi_{\text {before }}\right\rangle=\sum_{m, n} \bar{A}_{m n}\left|\alpha_{m}\right\rangle\left|\psi_{n}\right\rangle|\alpha\rangle,
$$

где

$$
|\alpha\rangle=\sum_{k} a_{k}\left|\alpha_{k}\right\rangle
$$

Теперь Алиса будет проводить измерение над системой $\mathbf{H} \otimes \mathbf{A}$, имеющее в данном ортонормированном базисе, скажем, вид

$$
\left|\beta_{j}\right\rangle=\sum_{r, s} C_{r s}^{j}\left|\psi_{r}\right\rangle\left|\alpha_{s}\right\rangle
$$

После измерения система $\mathbf{H} \otimes \mathbf{A}$ коллапсирует к одному из указанных выше состояний, скажем к $\left|\beta_{0}\right\rangle$. Таким образом, система $\widehat{\mathbf{H}}$ коллапсирует к состоянию

$$
\left|\Psi_{\text {after }}\right\rangle=\sum_{m, n, k} \bar{A}_{m n} C_{n k}^{0} a_{k}\left|\alpha_{m}\right\rangle=\bar{A} C^{0}|\alpha\rangle,
$$

где $\bar{A}$ - матрица с элементами $\bar{A}_{m n}$, а $C^{0}$ - матрица с элементами $C_{n k}^{0}$. Далее Алиса передает Бобу результат своего наблюдения (который на этот раз есть $\left|\beta_{0}\right\rangle$ ) 
по классическому каналу связи. На основе этого сообщения Боб будет знать, как выбрать $C^{0}$. Если бы $A$ было известно, Боб был бы в состоянии найти исходное квантовое состояние $|\alpha\rangle$. Действительно, все, что ему нужно сделать - это применить обратную матрицу (см. замечание в следующем абзаце) к $\left|\Psi_{\text {after }}\right\rangle$, т.е. $|\alpha\rangle=\left(C^{0}\right)^{-1} \bar{A}^{-1}\left|\Psi_{\text {after }}\right\rangle$.

Напомним, что оператор $A$ зависит от теории, которую мы здесь представили, и соотношение (43) можно использовать для ее проверки. Действительно, заметим, что $C^{0}|\alpha\rangle$ можно естественным образом отождествить с вектором из Н. Предполагая матрицу $C^{0}$ несингулярной (а в следующем разделе показано, что это допустимое предположение), получаем, что $C^{0}\left|\alpha_{k}\right\rangle, k=1,2, \ldots, N$, образуют базис в этом пространстве. Поэтому повторения эксперимента, когда выбраны $|\alpha\rangle=\left|\alpha_{k}\right\rangle$ для всех $k=1,2, \ldots, N$, приведут к полному заданию $A$, если только всякий раз имеется полный доступ к $\left|\Psi_{\text {after }}\right\rangle$. Естественно, это идеализированный сценарий - для определения $\left|\Psi_{\text {after }}\right\rangle$ с достаточной точностью требуются существенные дополнительные усилия и повторяющиеся акты эксперимента. В частности, следует также обеспечить взаимодействие системы $\widehat{\mathbf{H}}$ с внешним аппаратом. Дело в том, что оператор $K$ определяет характеристику телепортационного канала, и таким образом его можно определить экспериментально. Результат можно сравнить с теоретическим предсказанием из (34) или с более общей моделью (см. раздел 5).

В итоге телепортационный эксперимент с холловской системой $\widehat{\mathbf{H}} \otimes \mathbf{H} \otimes \mathbf{A}$ дает прямой доступ к динамической переменной рассматриваемой теории. Изучение свойств получающегося телепортационного канала должно, по крайней мере в принципе, позволить охарактеризовать матрицу $A$, а вслед за тем и оператор $K$. Более того, $K$ определяет $\rho=K^{*} K$. В частности, эксперимент определяет собственные значения оператора $\rho$, и результаты эксперимента можно сравнить с теоретическими предсказаниями. Если эксперимент подтверждает данную картину, и коль скоро матрица $A$ задана, то систему можно использовать в квантовом вычислительном устройстве.

\section{4. Технические предположения о производимом Алисой измерении.} Чтобы использовать телепортационный канал для проверки матрицы $A$ из (40), мы полагаем, что имеется ортонормированный базис в $\mathbf{H} \otimes \mathbf{A}$, заданный в виде (42), где, кроме того, $C^{j}$ несингулярны. Напомним наше допущение, что размерности пространств Н и $\mathbf{A}$ совпадают и равны $N$, и мы отождествляем эти два пространства, т.е. $\mathbf{A} \cong \mathbf{H}$. Это устанавливает отождествление пространства $\mathbf{H} \otimes \mathbf{A}$ с пространством $(N \times N)$-матриц. Подходящие семейства обратимых матриц $C^{j}$ были построены в работе [9], где было показано, что существуют ортонормированные базисы в пространстве матриц, состоящие из (нормированных) унитарных матриц.

Сохраняя те же обозначения, что и выше, опишем конструкцию следующим образом. Пусть $V$ - унитарный оператор циклического сдвига: $\left\langle\alpha_{k}\right| V=\left\langle\alpha_{k+1}\right|$, $k=1, \ldots, N$, причем $\left\langle\alpha_{N+1}\right|=\left\langle\alpha_{1}\right|$. Известно, что собственные значения оператоpa $V$ являются корнями из единицы, $u_{k}=e^{2 \pi i k / N}$, а соответствующие собственные векторы $\left\langle u_{k}\right|, k=1, \ldots, N$, образуют ортонормированный базис (в $\left.\mathbf{H}\right)$. С небольшой модифицикацией конструкция повторяется следующим образом: строится такой унитарный оператор циклического сдвига $U$, что $\left\langle u_{k}\right| U^{-1}=\left\langle u_{k+1}\right|, k=1, \ldots, N$. Тогда можно видеть, что набор $C^{m n}=N^{-1 / 2} U^{m} V^{n}, m, n=0, \ldots, N-1$, является 
ортонормированным базисом в пространстве $(N \times N)$-матриц. Он состоит из унитарных матриц (подвергнутых должному изменению масштаба). Заметим, что мы заменили индекс $j$ на более естественный двойной индекс $(m, n)$.

\section{5. ИСПОЛЬЗОВАНИЕ КВАНТОВОЙ ТЕЛЕПОРТАЦИИ ДЛЯ ОПРЕДЕЛЕНИЯ МОДЕЛИ ВЗАИМОДЕЙСТВИЯ ЭЛЕКТРОННОГО ГАЗА}

Анализ, представленный в предыдущем разделе, служит как минимум двум различным целям. Во-первых, он подсказывает, что квантовая холловская система представляет собой некий тип канала квантовой телепортации. Этот факт можно проверить только в эксперименте. Во-вторых, и это сейчас будет главным предметом нашего внимания, представленный выше формализм актуален для систем более общего типа. В частности, общий подход может быть актуален в применении к квантовой холловской системе, действующей в ином режиме, нежели режим KЭХ, например когда изменение температуры или других параметров заставляет систему выпасть из фазы КЭХ. Приведем некоторые рассуждения о том, как можно экспериментально оценить конкретный тип взаимодействия электронного газа в заданном режиме. Для этого взглянем еще раз на функционал $\Xi$ из (26). Как мы уже отмечали, его можно записать в виде

$$
\Xi(K)=\operatorname{Tr}\left(K H K^{*}\right)+\beta \operatorname{Tr} \ln \left(K^{*} K\right) .
$$

Рассмотрим обобщение этого функционала, а именно,

$$
\Xi_{f, \rho^{0}}(K)=\operatorname{Tr}\left(K H K^{*}\right)+\beta \operatorname{Tr}\left(\rho^{0} f\left(K^{*} K\right)\right),
$$

где $f$ - аналитическая функция. В наших обозначениях

$$
\Xi_{f, \rho^{0}}(K)=\operatorname{Tr}(H \rho)+\beta \operatorname{Tr}\left(\rho^{0} f(\rho)\right) .
$$

Пусть соответствующие собственные значения оператора $\rho$ равны $\rho_{1}, \rho_{2}, \ldots, \rho_{N}$. Как и раньше, мы предполагаем, что $\rho$ имеет обратный оператор и что $\operatorname{Tr} \rho=\rho_{1}+\rho_{2}+\cdots$ $\cdots+\rho_{N}=1$. Поэтому собственные значения лежат внутри единичного круга и на положительной полуоси $x$. Мы предполагаем, что $f(z)$ аналитична в единичном круге и принимает вещественные значения при вещественных $z=x$. Функцию $f(\rho)$ можно задать интегралом Коши

$$
f(\rho)=\frac{1}{2 \pi i} \int_{\Gamma} f(\zeta)(\zeta I-\rho)^{-1} d \zeta,
$$

где кривая Г ограничивает некоторую область в единичном круге, содержащую все собственные значения оператора $\rho$. Поскольку оператор $\rho$ не фиксирован, нам надо убедиться, что Г годится для всех возможных распределений собственных значений, хорошим выбором для Г является единичная окружность. В этом случае следует предполагать, что $f$ представима интегралом Коши от граничных значений. В наиболее общих терминах это имеет место, если $f$ принадлежит классу Харди $H^{1}[10]$. Далее, вычисление показывает, что

$$
D f(\rho)\left[\rho^{0}\right]=\left.\frac{d}{d \varepsilon}\right|_{\varepsilon=0} f\left(\rho+\varepsilon \rho^{0}\right)=\frac{1}{2 \pi i} \int_{\Gamma} f(\zeta)(\zeta I-\rho)^{-1} \rho^{0}(\zeta I-\rho)^{-1} d \zeta .
$$


Используя эту формулу, получаем

$$
\begin{aligned}
& \left.\frac{d}{d \varepsilon}\right|_{\varepsilon=0} \operatorname{Tr}\left[\rho^{0} f\left(\left(K^{*}+\varepsilon \Phi^{*}\right)(K+\varepsilon \Phi)\right)\right]=\left\langle K^{*} \Phi+\Phi^{*} K \mid D f(\rho)\left[\rho^{0}\right]\right\rangle= \\
& =\left\langle\Phi \mid K D f(\rho)\left[\rho^{0}\right]\right\rangle+\left\langle\Phi^{*} \mid D f(\rho)\left[\rho^{0}\right] K^{*}\right\rangle=2 \omega\left(\Phi,-i K D f(\rho)\left[\rho^{0}\right]\right) .
\end{aligned}
$$

Заметим, что эта формула является обобщением формулы (27).

Теперь можно записать аналог уравнения стационарных состояний (30) для случая обобщенного мезоскопического гамильтониана (45). Получаем

$$
K H+\beta K D f(\rho)\left[\rho^{0}\right]=\nu K .
$$

Поскольку $K$ несингулярен, отсюда следует, что

$$
D f(\rho)\left[\rho^{0}\right]=\frac{1}{\beta}(\nu I-H)
$$

Таким образом, оператор запутывания $K$ принимает вид $K=U \rho^{1 / 2}$, где по-прежнему $U: \mathbf{H} \rightarrow \widehat{\mathbf{H}}$ - произвольный унитарный оператор, а $\rho$ - решение уравнения (50). В действительности априори не гарантировано, что решение существует при любом выборе $\rho^{0}$. Рассмотрим уравнение (50) в локальных координатах. Удобно описывать оператор $D=D f(\rho)\left[\rho^{0}\right]$ в базисе собственных функций для $\rho$. Формула Коши (48) дает

$$
D_{j k}=\left\{\begin{array}{lll}
\rho_{j k}^{0} \frac{f\left(\rho_{j}\right)-f\left(\rho_{k}\right)}{\rho_{j}-\rho_{k}}, & \text { если } & \rho_{j} \neq \rho_{k}, \\
\rho_{j k}^{0} f^{\prime}\left(\rho_{k}\right), & \text { если } & \rho_{j}=\rho_{k} .
\end{array}\right.
$$

Будем считать для простоты, что $\rho_{1}<\rho_{2}<\cdots<\rho_{N}$. Тогда уравнение (50) эквивалентно уравнениям

$$
\rho_{j k}^{0} \frac{f\left(\rho_{j}\right)-f\left(\rho_{k}\right)}{\rho_{j}-\rho_{k}}=-\frac{H_{j k}}{\beta}, \quad j \neq k, \quad \rho_{k k}^{0} f^{\prime}\left(\rho_{k}\right)=\frac{\nu-H_{k k}}{\beta} .
$$

Заметим, что эта система уравнений для $\rho_{k}$ может оказаться переопределенной или противоречивой в зависимости от $\rho_{j k}^{0}$ и $H_{j k}$. Действительно, если $f^{\prime}(\cdot)-$ взаимно однозначная функция (например, $f(\rho)=\ln \rho$ ), то второе уравнение в (52) уже само по себе определяет все собственные значения $\rho_{k}$, а первые, внедиагональные, уравнения можно рассматривать как ограничения на $\rho_{j k}^{0}$. Подчеркнем, однако, что в данном разделе принята иная точка зрения. А именно, будем считать, что $\rho_{j k}, \rho_{j k}^{0}$ и $H_{j k}$ известны, например, из эксперимента. В частности, предположим, что $\rho_{j k}$ определяются из телепортационного эксперимента, описанного в разделе 4 . Соотношения (52) можно использовать для восстановления модели взаимодействия $f$. Пусть, например, $\rho^{0}=\bar{I}$, так что $\rho_{k k}^{0}=1 / N$. Уравнения (52) будут выполнены, только если все внедиагональные энергетические члены обращаются в нуль, т.е. $H_{j k}=0$. Кроме того, если потребовать, чтобы $f(\cdot)$ была возрастающей функцией, т.е. $f^{\prime}(\rho)>0$, то с необходимостью $\beta\left(\nu-H_{k k}\right)>0$ для всех $k$, что приводит к дальнейшим ограничениям на модель. Однако если все необходимые условия существования решений выполнены, то соотношение $f^{\prime}\left(\rho_{k}\right)=N\left(\nu-H_{k k}\right) / \beta$ позволяет 
частично определить $f$. Если $\rho_{k}$ и $H_{k k}, \nu$ и $\beta$ известны, а описанный в предыдущих разделах телепортационный эксперимент действительно определяет все $\rho_{k}$, то приведенное выше уравнение представляет собой задачу экстраполяции, которая ограничивает допустимые способы выбора функции $f(\cdot)$. Напомним, что $f$ идентифицирует систему посредством функционала (45). Таким образом, телепортационный эксперимент дает возможность получать информацию о статистической модели системы Н в том смысле, что он помогает определить $f$.

\section{Список литературы}

[1] A. Sowa, J. Phys. Chem. Solids, 65:8-9 (2004), 1507-1515.

[2] A. Sowa, J. Geom. Phys., 55:1 (2005), 1-18.

[3] A. Sowa, Russ. J. Math. Phys., 15:1 (2008), 122-127.

[4] A. Sowa, Adv. Stud. Theor. Phys., 1:9-12 (2007), 433-448.

[5] A. Sowa, Adv. Math. Phys., 2009 (2009), Art. ID 514081.

[6] В. И. Арнольд, Математические методы классической механики, Наука, М., 1974.

[7] S. Stenholm, K.-A. Suominen, Quantum Approach to Informatics, Wiley, Hoboken, NJ, 2005.

[8] V.P. Gusynin, V. A. Miransky, I. A. Shovkovy, Phys. Rev. D, 52:8 (1995), 4718-4735.

[9] J. Schwinger, Proc. Nat. Acad. Sci., 46:4 (1960), 570-579.

[10] А. Зигмунд, Тригонометрические рядь, Мир, М., 1965. 